\title{
Dynamic Output Feedback Control for Nonlinear Uncertain Systems with Multiple Time-Varying Delays
}

\author{
Wei Zheng ${ }^{1, ~ *}$, Hongbin Wang ${ }^{1}$, Zhiming Zhang ${ }^{2}$, Pengheng Yin ${ }^{1}$ \\ ${ }^{1}$ Institute of Electrical Engineering, Yanshan University, Qinhuangdao, China \\ ${ }^{2}$ China National Heavy Machinery Research Institute, Xi'an, China
}

Email address:

xiaoxiong19871110@163.com(Wei Zheng)

${ }^{*}$ Corresponding author

\section{To cite this article:}

Wei Zheng, Hongbin Wang, Zhiming Zhang, Pengheng Yin. Dynamic Output Feedback Control for Nonlinear Uncertain Systems with Multiple Time-Varying Delays. Automation, Control and Intelligent Systems. Vol. 6, No. 1, 2018, pp. 8-19. doi: 10.11648/j.acis.20180601.12

Received: December 26, 2017; Accepted: January 25, 2018; Published: April 2, 2018

\begin{abstract}
This paper addresses the adaptive dynamic output-feedback control problem for a class of nonlinear discrete-time systems with multiple time-varying delays. First, the guaranteed cost function is introduced for the nonlinear system to reduce the effect of the time-varying delays. Secondly, in order to deal with the multiple time-varying delays, the nonlinear system is decomposed into two subsystems. Then the compensator is designed for the first subsystem, and the adaptive dynamic output-feedback controller is constructed based on the subsystems. By introducing the new discrete Lyapunov-Krasovskii functional, it can be seen that the solutions of the resultant closed-loop system converge to an adjustable bounded region. Finally, the simulations are performed to show the effectiveness of the proposed methods.
\end{abstract}

Keywords: Multiple Time-Varying Delays, Parametric Uncertainties, Dynamic Output Feedback Control, Lyapunov-Krasovskii Functional

\section{Introduction}

Many practical systems are the nonlinear systems and consist of time-delay, such as the urban traffic networks system, digital communication system, and the power systems [1-3]. As universal approximators, fuzzy systems or neural networks have been successfully applied to solve the control design problem for various kinds of such nonlinear systems, and many interesting results have been obtained see [4-6] and the references therein. It is noted that with those control schemes, the stability of the closed-loop systems can be guaranteed, and the tracking errors can be confined to a small residual set, while the size of the residual sets is often unknown, and the transient and/or steady state performance cannot be prescribed.

The Lyapunov-Krasovskii functional method and Lyapunov-Razumikhin method are always employed for the system design. In recent years, many adaptive fuzzy/neural output-feedback control approaches have been proposed for uncertain SISO/MIMO nonlinear systems with unmeasured states in $[7,8]$. Note that all the aforementioned adaptive fuzzy/neural output-feedback control schemes are for the nonlinear strict-feedback uncertain systems [9], instead of the nonlinear nonstrict-feedback ones [10, 11]. In [12], the robust-control problem for the time-delay systems was considered, and the nonlinear uncertainties are bounded by the high-order polynomials. A tracking control system has a more general form than a general nonlinear system, and yet the system functions contain the whole state variables [13, 14]. But how to apply this method into the nonlinear time-delay systems is a challenging subject [15]. By employing the neural network technique, the state feedback controller is designed for the time-delay nonlinear systems. Compared with the previous work, in the effort to develop new adaptive control strategies, adaptive neural back-stepping state feedback control methodologies for nonlinear systems were proposed in [18]. There are few results on dynamic output feedback control for nonlinear system with time delays

It has been early recognized that the multiple time-varying characteristic deserve further research. In practice, time delay is one of the most important problems which usually appears in many industrial control systems [19]. For single nonlinear system, the time delay had been investigated in many references [20]. Recently, some static output-feedback control 
methods were proposed for the robotic system.

Based on the neural networks, a robust adaptive control method for a class of uncertain nonlinear systems in the presence of input saturation and external disturbance was proposed [21]. And an adaptive tracking control was designed for a class of uncertain multi-input and multi-output nonlinear systems with non-symmetric input constraints by using an auxiliary system [22]. Then, the input saturation problem was investigated for stochastic nonlinear systems [23]. In [24], the output feedback control problem for identical linear dynamic systems with input saturation was addressed. To our best knowledge, there are no results on output feedback control results for feedback nonlinear systems with time-delays and multiple subsystems.

In this paper, the dynamic output feedback control approach is proposed for the mobile robot system with multiple time-varying delays. The contributions of this paper can be summarized as follows:

(1) The cost function is introduces to deal with the time-delays.

(2) The nonlinear system is decomposed into two subsystems based on the input matrix and output matrix. The dynamic compensator is developed for the first subsystem. And the dynamic output-feedback controller is designed based on the second subsystem.

(3) By introducing the new Lyapunov-Krasovskii functional, it can be seen that the solutions of the resultant closed-loop system converge to an adjustable bounded region.

This paper is organized as follows. The preliminary knowledge for the nonlinear multiple time-varying delays system with parametric uncertainties are described in Section 2. The dynamic output-feedback controller for the nonlinear system is designed in Section 3. The results are further extended to the general nonlinear discrete-time case in Section 4. The simulation results are performed for a mobile robot case in Section 5. Finally, Section 6 concludes with a summary of the obtained results.

The rest of paper is organized as follows. Section 2 presents some preliminary knowledge for the dynamicsystem with. In

Section 3, the dynamic output-feedback controller is presented. The proving process is shown in Section 4. The simulation results are presented in Section 5. Finally, Section 6 concludes with a summary of the obtained results

\section{System Formulation and Preliminaries}

Consider a nonlinear discrete system with multiple time-varying delays and parametric uncertainties as follows.

$$
\left\{\begin{aligned}
& x(k+1)= \sum_{j=0}^{r}\left(A_{j}+\Delta A_{j}\right) x\left(k-\tau_{j}(k)\right)+B(u(k) \\
&\left.+f\left(x(k), x\left(k-\tau_{1}(k)\right), x\left(k-\tau_{2}(k)\right), \ldots, x\left(k-\tau_{r}(k)\right)\right)\right) \\
& y(k)=C x(k)
\end{aligned}\right.
$$

where $x(k) \in R^{n}$ is the state variable, $u(k) \in R^{m}$ is the control input, and $y(k) \in R^{p}$ is the control output. $A_{j} \in R^{n \times n}$,
$B \in R^{n \times m}$ and $C \in R^{p \times n}$ are gain matrices with appropriate dimensions, $\Delta A_{j} \in R^{n \times n}$ are the unknown matrix representing the parametric uncertainties. $\tau_{j}(k)$ are the multiple time-varying delays satisfies $1>\tau_{j}^{*} \geq \tau_{j}(k+1)$ and $\bar{\tau}_{j} \geq \tau_{j}(k)$, where $\tau_{0}(k)=0, \tau_{j}^{*}$ and $\bar{\tau}_{j}$ are the positive scalars. The nonlinear function $f(\bullet)$ is uncertain and contains multiple time-varying delays.

Assumption 1. For the nonlinear function $f(\bullet)$, the following inequality holds

$$
\begin{aligned}
& \sum_{j=0}^{r} \vartheta_{j}^{T} \alpha_{j}\left(\left\|x\left(k-\tau_{j}(k)\right)\right\|\right) \\
& \geq\left\|f\left(x(k), x\left(k-\tau_{1}(k)\right), x\left(k-\tau_{2}(k)\right), \ldots, x\left(k-\tau_{r}(k)\right)\right)\right\|
\end{aligned}
$$

where $\vartheta_{j} \in R^{p j} \quad$ is unknown constant vector. $\alpha_{j}(\bullet)=\left[\alpha_{j 1}(\bullet), \alpha_{j 2}(\bullet), \ldots, \alpha_{j i}(\bullet)\right]^{T}, \alpha_{j i}$ is known function with $\alpha_{j i}(0)=0$. And there exist the nonlinear functions $\bar{\alpha}_{j i}(\bullet)$ satisfying $x \bar{\alpha}_{j i}(x) \geq \alpha_{j i}(x)$.

Remark 1. In this paper, the adaptive control theory was presented to estimate the system parameters or uncertain bound parameters. In [25], the adaptive control theory was discussed with some unknown interconnections. Since the research contains the nonlinear functions, it can be seen that the schemes are not enforceable to system (1). In this paper, there are three challenging problems as follows: how to design a dynamic compensator with the output feedback signal; how to reduce the influence of uncertain parameters; and how to design the adaptive output feedback controller for the multiple time-delays system. The aim of this paper is to solve the above issues, and then the controller will be easy to implement in practical systems.

In this paper, $B=\left[\begin{array}{ll}0_{(n-m) \times m} & \bar{B}_{m \times m}\end{array}\right]^{T}$ and $C=\left[\begin{array}{ll}0_{(n-p) \times p} & D_{p \times p}\end{array}\right]^{T}$, where $\quad \bar{B}_{m \times m}$ and $D$ satisfying $\operatorname{Rank}\left(\bar{B}_{m \times m}\right)=m$ and $D D^{T}=D^{T} D=\mathrm{U}_{\mathrm{m}}, \mathrm{U}_{\mathrm{m}}$ is the identity matrix. Let $x=\left[\begin{array}{l}x_{1}^{T} \\ x_{2}^{T}\end{array}\right]$, then the system (1) is rewritten as follows:

$$
\left\{\begin{aligned}
x_{1}(k+1)= & \sum_{j=0}^{r}\left(\left(A_{j 12}+\Delta A_{j 12}\right) x_{2}\left(k-\tau_{j}(k)\right)\right. \\
& \left.+\left(A_{j 11}+\Delta A_{j 11}\right) x_{1}\left(k-\tau_{j}(k)\right)\right) \\
x_{2}(k+1) & =\sum_{j=0}^{r}\left(\left(A_{j 22}+\Delta A_{j 22}\right) x_{2}\left(k-\tau_{j}(k)\right)\right. \\
& \left.+\left(A_{j 21}+\Delta A_{j 21}\right) x_{1}\left(k-\tau_{j}(k)\right)\right) \\
& +\bar{B} f\left(x(k), x\left(t-\tau_{1}(k)\right), \ldots, x\left(k-\tau_{r}(k)\right)\right)+\bar{B} u(k) \\
y(k)=C & {\left[\begin{array}{c}
x_{1}^{T} \\
x_{2}^{T}
\end{array}\right] }
\end{aligned}\right.
$$

where $A_{j 11}, A_{j 12}, A_{j 21}$ and $A_{j 22}$ are the decomposition 
matrices of $A_{j} . \Delta A_{j 11}, \Delta A_{j 12}, \Delta A_{j 21}$ and $\Delta A_{j 22}$ are the decomposition matrix of $\Delta A_{j}$. With $m<p$, one has $y=\left[\begin{array}{l}y_{1}^{T} \\ y_{2}^{T}\end{array}\right]$. For the matrices $B$ and $C$, there exist the nonsingular matrices $E \in R^{m \times m}$ and $\tilde{C} \in R^{(p-m)(n-m)}$ satisfying $y_{1}=\tilde{C} x_{1}$ and $y_{2}=E x_{2}$.

With the above analysis, the system (1) is further rewritten as follows:

$$
\left\{\begin{aligned}
& x_{1}(k+1)= \sum_{j=0}^{r}\left(\left(\bar{A}_{j 11}+\Delta \bar{A}_{j 11}\right) x_{1}\left(k-\tau_{j}(k)\right)\right. \\
&\left.+\left(\bar{A}_{j 12}+\Delta \bar{A}_{j 12}\right) y_{2}\left(k-\tau_{j}(k)\right)\right) \\
& y_{2}(k+1)= \sum_{j=0}^{r}\left(\left(\bar{A}_{j 21}+\Delta \bar{A}_{j 21}\right) x_{1}\left(k-\tau_{j}(k)\right)\right. \\
&\left.+\left(\bar{A}_{j 22}+\Delta \bar{A}_{j 22}\right) y_{2}\left(k-\tau_{j}(k)\right)\right) \\
&+E \bar{B} f\left(x(k), x\left(k-\tau_{1}(k)\right), \ldots, x\left(k-\tau_{r}(k)\right)\right)+E \bar{B} u(k) \\
& y(k)=\left[\begin{array}{c}
\left(\tilde{C} x_{1}\right)^{T} \\
\left(E x_{2}\right)^{T}
\end{array}\right]
\end{aligned}\right.
$$

where $\quad \bar{A}_{j 11}=A_{j 11} \quad, \quad \bar{A}_{j 12}=A_{j 12} E^{-1} \quad, \quad \bar{A}_{j 21}=E A_{j 21} \quad$, $\bar{A}_{j 22}=E A_{j 22} E^{-1} \quad, \quad \Delta \bar{A}_{j 11}=\Delta A_{j 11} \quad, \quad \Delta \bar{A}_{j 12}=\Delta A_{j 12} E^{-1}$ ， $\Delta \bar{A}_{j 21}=E \Delta A_{j 21}$ and $\Delta \bar{A}_{j 22}=E \Delta A_{j 22} E^{-1}$.

\section{Controller Design}

First, a cost function is introduced for the nonlinear system to deal with the uncertain parameters $\Delta A_{j}$. Secondly, a dynamic compensator is designed for the subsystem- $x_{1}$, and the adaptive output-feedback controller is constructed base on the second subsystem- $y_{2}$ and the compensator.

\subsection{Cost Function Design}

In this study, the uncertainties are norm-bounded, described as follows:

$$
\left[\begin{array}{cc}
\Delta A_{j 11} & \Delta A_{j 12} \\
\Delta A_{j 21} & \Delta A_{j 22}
\end{array}\right]=M(k)\left[\begin{array}{ll}
Q_{1} & Q_{2}
\end{array}\right]
$$

where $Q_{1}$ and $Q_{2}$ are the constant matrices, $M(k)$ is an unknown matrix satisfying $M^{T}(k) M(k) \leq \mathrm{U}_{m}$. Let $0<\lambda_{1} \in R^{n \times n}$ and $0<\lambda_{2} \in R^{m \times m}$. Consider the cost function for the system (1) as follows

$$
J=\sum_{t=0}^{\infty}\left[x^{T}(k) \lambda_{1} x(k)+u^{T}(k) \lambda_{2} u(k)\right]
$$

Now, in order to improve the stability of system (3), one has

$$
\begin{aligned}
& \hat{x}(k+1)=A_{c} \hat{x}(k)+B_{c} y(k), \quad \hat{x}(0)=0 \\
& u(k)=C_{c} \hat{x}(k)
\end{aligned}
$$

where $\hat{x}(k)$ is the state vector. Then applying (6) to the system (3), the new closed-loop system is obtained as follows

$$
\bar{x}(k+1)=\left(\bar{A}_{j}+M(k) \bar{Q}\right) \bar{x}(k)+\sum_{j=0}^{r}\left(\bar{A}_{j}+M(k) Q_{2}\right) x\left(k-\tau_{j}(k)\right)
$$

where

$$
\bar{x}(k)=\left[\begin{array}{l}
x(k) \\
\hat{x}(k)
\end{array}\right], \bar{A}_{j}=\left[\begin{array}{cc}
A_{j} & B C_{c} \\
B_{c} C & A_{c}
\end{array}\right], \bar{Q}=\left[\begin{array}{ll}
Q_{1} & Q_{2} C_{c}
\end{array}\right]
$$

For the closed-loop system (7), design the cost function as follows

$$
J=\sum_{t=0}^{\infty} \bar{x}^{T}(k) \bar{\lambda} \bar{x}(k)
$$

where $\bar{\lambda}=\operatorname{diag}\left[\lambda_{1}, C_{c}^{T} \lambda_{2} C_{c}\right]$.

With the cost function (5), the Assumption 2 is imposed on system (3):

Assumption 2. Consider the nonlinear system (3) and cost function (5), the initial states of system (3) are given. If there exist the equation (6) and a positive scalar $J^{*}$ such that $J \leq J^{*}$, then the closed-loop system is stable.

Lemma 1. [26]: Let $\hat{J}=\hat{J}^{T}>0, \hat{m}_{1}, \hat{m}_{2}$ are the known matrices with appropriate dimensions. Then the following inequalities hold:

$$
\begin{gathered}
\hat{J}+\varpi^{-1} \hat{m}_{1} \hat{m}_{1}^{T}+\varpi \hat{m}_{2}^{T} \hat{m}_{2}<0 \\
\hat{J}+\hat{m}_{1} M \hat{m}_{2}+\hat{m}_{2}^{T} M^{T} \hat{m}_{1}^{T}<0
\end{gathered}
$$

where $M^{T} M \leq \mathrm{U}_{m}$ and $\varpi$ is a positive scalar.

Lemma 2. [27]: For matrices $n_{1}>0$ and $n_{2}>0$, if there exist a matrix $\Theta$ such that $\Theta=\Theta^{T}>0$, then the following inequality holds:

$$
-2 n_{1}^{T} n_{2} \leq n_{1}^{T} \Theta n_{1}+n_{2}^{T} \Theta^{-1} n_{2} .
$$

\subsection{Compensator Design}

For subsystem- $x_{1}(k+1)$, the augmented dynamic system is designed as follows:

$$
\left\{\begin{array}{l}
\bar{y}_{2}(k)=C_{l} \zeta(k)+D_{l} y_{1}(k) \\
\zeta(k+1)=A_{l} \zeta(k)+B_{l} y_{1}(k)
\end{array}\right.
$$

where $\zeta \in R^{n-m}, \bar{y}_{2}(k) \in R^{m}, A_{l}, B_{l}, C_{l}$ and $D_{l}$ are designed with appropriate dimensions. With equations (4) and (9), one has:

$$
\delta(k+1)=\sum_{j=1}^{r} N_{j} \delta\left(k-\tau_{j}(k)\right)+I \delta(k)+w
$$


where $w=\sum_{j=0}^{r} \bar{A}_{j 12} z\left(k-\tau_{j}(k)\right)$

in which

$$
z(k)=y_{2}(k)-\bar{y}_{2}(k), \delta(k)=\left[\begin{array}{c}
x_{1}(k) \\
\zeta(k)
\end{array}\right], I=\left[\begin{array}{cc}
\bar{A}_{011}+\bar{A}_{012} D_{l} \tilde{C} & \bar{A}_{012} C_{l} \\
B_{l} \tilde{C} & A_{l}
\end{array}\right] \text { and } N_{j}=\left[\begin{array}{cc}
\bar{A}_{j 11}+\bar{A}_{j 12} D_{l} \tilde{C} & \bar{A}_{j 12} C_{l} \\
0 & 0
\end{array}\right]
$$

For equation (10), choose a discrete Lyapunov-Krasovskii functional as follows:

$$
\begin{aligned}
V_{1} & =\sum_{j=1}^{r} \int_{k-\tau_{j}(k)}^{k} e^{c(\sigma-k)} \delta^{T}(\sigma) R_{j} \delta(\sigma) d \sigma \\
& +\delta^{T}(k) P \delta(k)+\sum_{j=1}^{r} \int_{-\bar{\tau}_{j}}^{0} \int_{k+\theta}^{k} e^{c(\sigma-k)} \dot{\delta}^{T}(\sigma) Z_{j} \dot{\delta}(\sigma) d \sigma d \vartheta
\end{aligned}
$$

where $P, R_{j}$, and $Z_{j}$ are the positive matrices, $c$ and $\sigma$ are the positive scalars. Now taking the forward difference of (11), one has:

$$
\begin{aligned}
\Delta V_{1} & \leq \sum_{j=1}^{r}\left(\bar{\tau}_{j} \delta^{T}(k+1) Z_{j} \delta(k+1)\right. \\
& \left.-\int_{k-\tau_{j}(k)}^{k} e^{-c \bar{\tau}_{j}} \dot{\delta}^{T}(\sigma) Z_{j} \dot{\delta}(\sigma) d \sigma\right) \\
& +\sum_{j=1}^{r}\left(\delta^{T}(k) R_{j} \delta(k)-\left(1-\tau_{j}^{*}\right)\right. \\
& \left.\times e^{-c \bar{\tau}_{j}} \delta^{T}\left(k-\tau_{j}(k)\right) R_{j} \delta\left(k-\tau_{j}(k)\right)\right) \\
& -c V_{1}+2 \delta^{T}(k) P \delta(k+1)+c \delta^{T}(k) P \delta(k)
\end{aligned}
$$

Note that:

$$
\begin{aligned}
& 2 \sum_{i=1}^{r}\left(\sum_{j=0}^{r} \delta^{T}\left(k-\tau_{j}(k)\right) \mathrm{T}_{i j}+w^{T}(k) \mathrm{T}_{i(r+1)}\right) \\
& \times\left(\delta(k)-\int_{k-\tau_{i}(k)}^{k} \dot{\delta}(\sigma) d \sigma-\delta\left(k-\tau_{i}(k)\right)\right)=0
\end{aligned}
$$

where $\mathrm{T}_{i j}$ is a weight matrix. With equations (10), (12) and (13), one has:

$$
\Delta V_{1} \leq \varepsilon\|w\|^{2}-c V_{1}+a^{T} G a+\sum_{i=1}^{r} \int_{k-\tau_{i}(k)}^{k} x^{T}(k, \zeta) F_{i} x(k, \zeta) d \zeta
$$

where $a$ and $\varepsilon$ are the positive scalars, $F_{i}=\left[\begin{array}{cc}X_{i} & \mathrm{~T}_{i} \\ \mathrm{~T}_{i}^{T} & -e^{-c \bar{\tau}_{i}} Z_{i}\end{array}\right]$ with $X_{i}=\left[X_{i l j}\right]_{(r+2) \times(r+2)}, \mathrm{T}_{i}=\left[\begin{array}{lll}-\mathrm{T}_{i 0}^{T} & \cdots & -\mathrm{T}_{i(r+1)}^{T}\end{array}\right]^{T} . G$ is defined as follows: $G_{11}=\sum_{i=1}^{r} \bar{\tau}_{i} I^{T} Z_{i} I+P I+\sum_{i=1}^{r}\left(\mathrm{~T}_{i 0}+\mathrm{T}_{i 0}{ }^{T}\right)+I^{T} P+c P-\sum_{i=1}^{r} \bar{\tau}_{i} X_{i 11}+\sum_{j=1}^{r} R_{j}$, $G_{1 j}=\sum_{i=1}^{r} \bar{\tau}_{i} T^{T} Z_{i} N_{j-1}+P N_{j-1}-\sum_{i=1}^{r} \bar{\tau}_{i} X_{i 1 j}-\mathrm{T}_{(j-1) 0}+\sum_{i=1}^{r} \mathrm{~T}_{i(j-1)}^{T} \quad$ for $r+1 \geq j \geq 2 ; \quad G_{1(r+2)}=\sum_{i=1}^{r} \mathrm{~T}_{i(r+1)}^{T}+\sum_{i=1}^{r} \bar{\tau}_{i} I^{T} Z_{i}-\sum_{i=1}^{r} \bar{\tau}_{i} X_{i 1(r+2)} P$, $G_{j j}=\sum_{i=1}^{r} \bar{\tau}_{i} N_{j-1}^{T} Z_{i} N_{j-1}-\sum_{i=1}^{r} \bar{\tau}_{i} X_{i j j}-\mathrm{T}_{(j-1)(j-1)}^{T}-e^{-c \bar{\tau}_{j-1}} R_{j-1}-\mathrm{T}_{(j-1)(j-1)}$ for $r+1 \geq j \geq 2$

$G_{j q}=\sum_{i=1}^{r} \bar{\tau}_{i} N_{j-1}^{T} Z_{i} N_{q-1}-\sum_{i=1}^{r} \bar{\tau}_{i} X_{i j q}-\mathrm{T}_{(j-1)(q-1)}^{T}-\mathrm{T}_{(q-1)(j-1)}$ for $r+1 \geq q \geq j+1 ; G_{j(r+2)}=-\sum_{i=1}^{r} \bar{\tau}_{i} X_{i j(r+2)}-\mathrm{T}_{(j-1)(r+1)}^{T}+\sum_{i=1}^{r} \bar{\tau}_{i} N_{j-1}^{T} Z_{i}$ for $r+1>j>2 ; G_{(r+2)(r+2)}=\sum_{i=1}^{r} \bar{\tau}_{i} Z_{i}-\varepsilon \mathrm{U}_{m}-\sum_{i=1}^{r} \bar{\tau}_{i} X_{i(r+2)(r+2)}$, $a=\left[\begin{array}{lllll}\delta^{T}(k) & \delta^{T}\left(k-\tau_{1}(k)\right) & \cdots & \delta^{T}\left(k-\tau_{r}(k)\right) & w^{T}\end{array}\right]^{T} \quad$, $x(k, \zeta)=\left[\begin{array}{cc}a^{T} & \dot{\delta}^{T}(\zeta)\end{array}\right]^{T}$ and $G=\left[G_{i j}\right]_{(r+2) \times(r+2)}$.

With the above analysis, the new results arise:

Theorem 1. $\forall$ positive scalar $\varepsilon$, there exist the positive matrices $P, R_{i}, Z_{i}, \mathrm{~T}_{i j}$ and $X_{i l j}$ such that $G<0$ and $F_{i}<0$ for any $i=(1,2, \ldots, r)$. With Theorem 1 and (14), taking the forward difference of $V_{1}$ along (10), one has

$$
\Delta V_{1} \leq \varepsilon\|w\|^{2}-c V_{1}
$$

Remark 2. The matrix $T_{i l}$ is employed to derive the conservative conditions. The use details of $T_{i l}$ is shown in $[28,29]$. The compensator $(n-m$ order $)$ is designed for subsystem- $x_{1}$. The matrices $A_{l}, B_{l}, C_{l}$, and $D_{l}$ are designed in (9), by letting $C_{l}=0$, the dynamic compensator (9) will become a static compensator.

Choose a new discrete Lyapunov-Krasovskii functional as follows:

$$
\bar{V}_{1}=V_{1}+\sum_{j=1}^{r}\left(1-\tau_{j}^{*}\right)^{-1} e^{c \bar{\tau}_{j}} \times \int_{k-\tau_{j}(k)}^{k} e^{c(\delta-k)} \varepsilon(1+r)\left\|\bar{A}_{j 12} z(\sigma)\right\|^{2} d \sigma
$$

with (15), one has:

$$
\Delta V_{1} \leq \sum_{j=0}^{r} \varepsilon(1+r)\left\|\bar{A}_{j 12} z\left(k-\tau_{j}(k)\right)\right\|^{2}-c V_{1}
$$

By direct verification, one can obtain

$$
\Delta \bar{V}_{1} \leq \bar{\varepsilon}\|z(k)\|^{2}-c \bar{V}_{1}
$$

where

$$
\bar{\varepsilon} \geq \varepsilon(1+r)\left\|\bar{A}_{012}\right\|^{2}+\sum_{j=1}^{r} \varepsilon\left(1-\tau_{j}^{*}\right)^{-1}(1+r) e^{c \bar{\tau}_{j}}\left\|\bar{A}_{j 12}\right\|^{2}
$$

With the above analysis and the compensator (9), the adaptive dynamic output-feedback controller will be designed in next section.

\subsection{Adaptive Dynamic Output-Feedback Controller Design}

Let

$$
\Lambda=\left[\begin{array}{ll}
C_{l} & D_{l} \tilde{C}
\end{array}\right] \quad, \quad \text { one can obtain }
$$


$z(k)=y_{2}(k)-\bar{y}_{2}(k)=y_{2}-\Lambda \delta(k)$. Since $\quad \alpha_{j i}(\bullet)$ is a unknown vector $\bar{\vartheta}_{j}$ such that nonlinear function, with Assumption 1, there exist the

$$
\begin{aligned}
\| & E \bar{B} f\left(x(k), x\left(k-\tau_{1}(k)\right), \ldots, x\left(k-\tau_{r}(k)\right)\right) \| \\
\leq & \sum_{j=0}^{r} \bar{\vartheta}_{j}^{T} \alpha_{j}\left(\left\|E^{-1} y_{2}\left(k-\tau_{j}(k)\right)\right\|+\left\|x_{1}\left(k-\tau_{j}(k)\right)\right\|\right) \\
\leq & \sum_{j=0}^{r} \bar{\vartheta}_{j}^{T} \alpha_{j}\left(\left\|x_{1}\left(k-\tau_{j}(k)\right)\right\|+\left\|E^{-1}\right\|\left\|z\left(k-\tau_{j}(k)\right)\right\|\right. \\
& \left.+\left\|E^{-1} \Lambda\right\| \delta\left(k-\tau_{j}(k)\right) \|\right) \\
\leq & \sum_{j=0}^{r} \bar{\vartheta}_{j}^{T}\left(\alpha_{j}\left(3\left\|x_{1}\left(k-\tau_{j}(k)\right)\right\|\right)+\alpha_{j}\left(3\left\|E^{-1}\right\|\left\|z\left(k-\tau_{j}(k)\right)\right\|\right)\right. \\
& \left.+\alpha_{j}\left(3\left\|E^{-1} \Lambda\right\|\left\|\delta\left(k-\tau_{j}(k)\right)\right\|\right)\right)
\end{aligned}
$$

With (4), one has:

$$
\begin{aligned}
z(k+1)= & \sum_{j=0}^{r}\left(\bar{A}_{j 22} y_{2}\left(k-\tau_{j}(k)\right)+\bar{A}_{j 21} x_{1}\left(k-\tau_{j}(k)\right)\right) \\
& +E \bar{B} u(k)-C_{l}\left(A_{l} \zeta(k)+B_{l} y_{1}(k)\right)-D_{l} \tilde{C} \dot{x}_{1} \\
& +E \bar{B} f\left(x(k), x\left(k-\tau_{1}(k)\right), \ldots, x\left(k-\tau_{r}(k)\right)\right)
\end{aligned}
$$

For subsystem- $z(k)$, where $z(k)=y_{2}(k)-\bar{y}_{2}(k)=y_{2}-\Lambda \delta(k)$, choose a discrete Lyapunov-Krasovskii functional as follows:

$$
\begin{aligned}
V_{2} & =\frac{1}{2 d}\left(\vartheta(k)-\vartheta^{*}\right)^{2}+z^{T}(k) z(k) \\
& +\sum_{j=1}^{r} e^{b \bar{\tau}_{j}}\left(1-\tau_{j}^{*}\right)^{-1} \times \int_{k-\tau_{j}(k)}^{k} e^{b(\sigma-k)} F_{j}\left(x_{1}(\sigma), \delta(\sigma), z(\sigma)\right) d \sigma
\end{aligned}
$$

where $\Lambda=\left[\begin{array}{ll}C_{l} & D_{l} \tilde{C}\end{array}\right], b$ and $d$ are the positive scalars, $\vartheta(k)$ is the adaptive parameter with $\vartheta^{*}=\sum_{j=0}^{r} 3 \Upsilon^{-1} \bar{\vartheta}_{j}^{T} \bar{\vartheta}_{j} . F_{j}(\bullet)$ is defined as:

$$
\begin{aligned}
& F_{j}\left(x_{1}(\sigma), \delta(\sigma), z(\sigma)\right) \\
= & \Upsilon\left\|\alpha_{j}\left(3\left\|E^{-1}\right\|\|z(\sigma)\|\right)\right\|^{2} \\
& \Upsilon \Upsilon\left\|\alpha_{j}\left(3\left\|E^{-1} \Lambda\right\|\|\delta(\sigma)\|\right)\right\|^{2}+\Upsilon\left\|\alpha\left(3\left\|x_{1}(\sigma)\right\|\right)\right\|^{2} \\
& +\mu_{1}^{-1}\left\|x_{1}(\sigma)\right\|^{2}+\mu_{2}^{-1}\|z(\sigma)\|^{2}+\mu_{2}^{-1}\left\|C_{l}\right\|^{2}\|\zeta(\sigma)\|^{2}
\end{aligned}
$$

where $\mu_{1} \mu_{2}$ and $\Upsilon$ are the positive scalars.

Now, taking the forward difference of $V_{2}$ yields:

$$
\begin{aligned}
\Delta V_{2} & \leq-b V_{2}+b z^{T}(k) z(k)+\frac{b}{2 d}\left(\vartheta(k)-\theta^{*}\right)^{2} \\
& +\frac{1}{d}\left(\vartheta(k)-\vartheta^{*}\right) \vartheta(k+1)+2 z^{T}(k) z(k+1) \\
& +\sum_{j=1}^{r}\left(\left(1-\tau_{j}^{*}\right)^{-1} e^{b \bar{\tau}_{j}} F_{j}\left(x_{1}(k), \delta(k), z(k)\right)\right. \\
& \left.-F_{j}\left(x_{1}\left(k-\tau_{j}(k)\right), \delta\left(k-\tau_{j}(k)\right), z\left(k-\tau_{j}(k)\right)\right)\right)
\end{aligned}
$$

With (18), one has:

$$
\begin{aligned}
& 2 z^{T}(k) z(k+1) \\
& =2 z^{T}(k)\left(E \bar{B} u(k)+E \bar{B} f-C_{l}\left(A_{l} \zeta(k)+B_{l} y_{1}(k)\right)\right) \\
& +2 z^{T}(k) \sum_{j=0}^{r}\left(\left(\bar{A}_{j 21}-D_{l} \tilde{C} \bar{A}_{j 11}\right) x_{1}\left(k-\tau_{j}(k)\right)\right. \\
& \left.+\left(\bar{A}_{j 22}-D_{l} \tilde{C} \bar{A}_{j 12}\right) y_{2}\left(k-\tau_{j}(k)\right)\right)
\end{aligned}
$$

By employing (17), such that:

$$
\begin{aligned}
& 2 z^{T}(k) E \bar{B} f\left(x(k), x\left(k-\tau_{1}(k)\right), \ldots, x\left(k-\tau_{r}(k)\right)\right) \\
& \leq 2\|z(k)\| \sum_{j=0}^{r} \bar{\vartheta}_{j}^{T}\left(\alpha_{j}\left(3\left\|x_{1}\left(k-\tau_{j}(k)\right)\right\|\right)\right. \\
& \left.+\alpha_{j}\left(3\left\|E^{-1} \Lambda\right\|\left\|\delta\left(k-\tau_{j}(k)\right)\right\|\right)+\alpha_{j}\left(3\left\|E^{-1}\right\|\left\|z\left(k-\tau_{j}(k)\right)\right\|\right)\right) \\
& \leq \sum_{j=0}^{r}\left(3 \Upsilon^{-1} \bar{\vartheta}_{j}^{T} \bar{\vartheta}_{j}\|z(k)\|^{2}+\Upsilon\left\|\alpha_{j}\left(3\left\|x_{1}\left(k-\tau_{j}(k)\right)\right\|\right)\right\|^{2}\right. \\
& +\Upsilon\left\|\alpha_{j}\left(3\left\|E^{-1} \Lambda\right\| \delta \delta\left(k-\tau_{j}(k)\right) \|\right)\right\|^{2} \\
& \left.+\Upsilon\left\|\alpha_{j}\left(3\left\|E^{-1}\right\|\left\|z\left(k-\tau_{j}(k)\right)\right\|\right)\right\|^{2}\right)
\end{aligned}
$$




$$
\begin{aligned}
& 2 z^{T}(k) \sum_{j=0}^{r}\left(\left(\bar{A}_{j 21}-D_{l} \tilde{C} \bar{A}_{j 11}\right) x_{1}\left(k-\tau_{j}(k)\right)\right. \\
& \left.+\left(\bar{A}_{j 22}-D_{l} \tilde{C} \bar{A}_{j 12}\right) y_{2}\left(k-\tau_{j}(k)\right)\right) \\
& =2 z^{T}(k) \sum_{j=0}^{r}\left(\bar{A}_{j 21}-D_{l} \tilde{C} \bar{A}_{j 11}\right. \\
& \left.+\left(\bar{A}_{j 22}-D_{l} \tilde{C} \bar{A}_{j 12}\right) D_{l} \tilde{C}\right) x_{1}\left(k-\tau_{j}(k)\right) \\
& +2 z^{T}(k) \sum_{j=0}^{r}\left(\bar{A}_{j 22}-D_{l} \tilde{C} \bar{A}_{j 12}\right) \times\left(z\left(k-\tau_{j}(k)\right)+C_{l} \zeta\left(k-\tau_{j}(k)\right)\right)
\end{aligned}
$$

By letting

$$
\begin{gathered}
\left\|\bar{A}_{j 21}+\left(\bar{A}_{j 22}-D_{l} \tilde{C} \bar{A}_{j 12}\right) D_{l} \tilde{C}-D_{l} \tilde{C} \bar{A}_{j 11}\right\|^{2} \leq s_{1} \\
\left\|\bar{A}_{j 22}-D_{l} \tilde{C} \bar{A}_{j 12}\right\|^{2} \leq s_{2}
\end{gathered}
$$

where $s_{1}$ and $s_{2}$ are the transition parameters.

For $j \in[1, r]$, one has:

$$
\begin{aligned}
& \mu_{1}^{-1}\left\|x_{1}(k)\right\|^{2}+(1+r) \mu_{1} s_{1}\|z(k)\|^{2}+\sum_{j=1}^{r} \mu_{1}^{-1}\left\|x_{1}\left(k-\tau_{j}(k)\right)\right\|^{2} \\
& \geq 2 z^{T}(k) \sum_{j=0}^{r}\left(\bar{A}_{j 21}-D_{l} \tilde{C} \bar{A}_{j 11}+\left(\bar{A}_{j 22}-D_{l} \tilde{C} \bar{A}_{j 12}\right) D_{l} \tilde{C}\right) x_{1}\left(k-\tau_{j}(k)\right)
\end{aligned}
$$

and

$$
\begin{aligned}
& \sum_{j=1}^{r}\left(\mu_{2}^{-1}\left\|z\left(k-\tau_{j}(k)\right)\right\|^{2}+\mu_{2}^{-1}\left\|C_{l}\right\|^{2}\left\|\zeta\left(k-\tau_{j}(k)\right)\right\|^{2}\right)+2 r \mu_{2} s_{2}\|z(k)\|^{2} \\
& \geq 2 z^{T}(k) \sum_{j=1}^{r}\left(\bar{A}_{j 22}-D_{l} \tilde{C} \bar{A}_{j 12}\right)\left(C_{l} \zeta\left(k-\tau_{j}(k)\right)+z\left(k-\tau_{j}(k)\right)\right)
\end{aligned}
$$

Design the control law $u(k)=(E \bar{B})^{-1} \bar{u}(k)$ for system (1) where

$$
\begin{aligned}
\bar{u}(k) & =\left(D_{l} \tilde{C}_{012}-\bar{A}_{022}\right)\left(z(k)+C_{l} \zeta(k)\right) \\
& +C_{l}\left(A_{l} \zeta(k)+B_{l} y_{1}(k)\right)-\frac{1}{2} \phi(z(k)) \\
& -\frac{1}{2} \vartheta(k) z(k)-\frac{1}{2}\left((r+1) \mu_{1} s_{1}+b\right. \\
& \left.+2 r \mu_{2} s_{2}+\sum_{j=1}^{r}\left(1-\tau_{j}^{*}\right)^{-1} e^{b \bar{\tau}_{j}} \mu_{2}^{-1}\right) z(k) \\
& -4.5 \Upsilon\left\|E^{-2}\right\| z(k)\left(\| \bar{\alpha}_{0}\left(3 \| E^{-1\|\| z(k) \|) \|^{2}}\right.\right. \\
& +\sum_{j=1}^{r}\left(1-\tau_{j}^{*}\right)^{-1} e^{b \bar{\tau}_{j}} \| \bar{\alpha}_{j}\left(3 \| E^{\left.-1\|\| z(k) \|) \|^{2}\right)}\right.
\end{aligned}
$$

in which $\phi(z(k))$ is a nonlinear function, $\phi(z(k))$ is employed to reduce the influences of parameter $\delta$ in subsystem (10).

Substituting (21)-(26) into (20) yields:

$$
\begin{aligned}
& \Delta V_{2} \leq \frac{1}{d}\left(\vartheta(k)-\vartheta^{*}\right) \vartheta(k+1)-b V_{2} \\
& +\left(\vartheta^{*}-\vartheta(k)\right) z^{T}(k) z(k)-z^{T}(k) \phi(z(k)) \\
& +\Phi(\delta(k))+\frac{b}{2 d}\left(\vartheta(k)-\vartheta^{*}\right)^{2}
\end{aligned}
$$

where $\Phi(\delta(k))$ is defined as follows:

$$
\begin{aligned}
\Phi(\delta(k))= & \mu_{1}^{-1}\left\|x_{1}(k)\right\|^{2}+\Upsilon\left\|\alpha_{0}\left(3\left\|x_{1}(k)\right\|\right)\right\|^{2} \\
& +\Upsilon\left\|\alpha_{0}\left(3\|\| E^{-1} \Lambda\|\| \delta(k)\|\|\right)\right\|^{2} \\
& +\sum_{j=1}^{r}\left(1-\tau_{j}^{*}\right)^{-1} e^{b \bar{\tau}_{j}} \Upsilon\left(\left\|\alpha_{j}\left(3\left\|x_{1}(k)\right\|\right)\right\|^{2}\right. \\
& \left.+\left\|\alpha_{j}\left(3\left\|E^{-1} \Lambda\right\|\|\delta(k)\|\right)\right\|^{2}\right) \\
& +\sum_{j=1}^{r}\left(1-\tau_{j}^{*}\right)^{-1} e^{b \bar{\tau}_{j}}\left(\mu_{2}^{-1}\left\|C_{l}\right\|^{2}\|\zeta(k)\|^{2}+\mu_{1}^{-1}\left\|x_{1}(k)\right\|^{2}\right)
\end{aligned}
$$

With the above analysis, the Theorem 2 . is given as follows Theorem 2. For system (1) with $u(k)=(E \bar{B})^{-1} \bar{u}(k), \bar{u}(k)$ is defined in (26). Defining $\phi(z(k))=\bar{\varepsilon} W\left(\frac{\bar{\varepsilon}}{c-a-\mu}\|z(k)\|^{2}\right) z(k)$ with $a+\mu<c$, there exists an increasing positive function $W(\bullet)$ satisfying (30) below, the adaptive law is defined as follows:

$$
\vartheta(k+1)=-\sigma d \vartheta(k)+d\|z(k)\|^{2}
$$

where $\sigma$ and $d$ are the positive scalars such that $d \sigma-b>0$, then the solutions of the closed-loop system convergent to a ball.

Proof. Choose the discrete Lyapunov-Krasovskii functional (29) for the system (4) as follows:

$$
V=V_{2}+\int{ }_{0}^{\bar{V}_{1}} W(\sigma) d \sigma
$$

where $W(\bullet)$ is an increasing positive function, such that: 


$$
\Phi(\delta(k)) \leq \mu \delta^{T}(k) P \delta(k) W\left(\delta^{T}(k) P \delta(k)\right)
$$

$\int_{0}^{\bar{V}_{1}} W(\sigma) d \sigma$ is employed to deal with the nonlinear function $\Phi(\delta(k))$. Then, taking the forward difference of $V$ yields

$$
\begin{aligned}
\Delta V & =W\left(\bar{V}_{1}\right) \Delta \bar{V}_{1}+\Delta V_{2} \\
& \leq-b V_{2}-z^{T}(k) \phi(z(k))+\frac{b}{2 d}\left(\vartheta(k)-\vartheta^{*}\right)^{2} \\
& +\left(\vartheta^{*}-\vartheta(k)\right) z^{T}(k) z(k)+\frac{1}{d}\left(\vartheta(k)-\vartheta^{*}\right) \vartheta(k+1) \\
& +\Phi(\delta(k))+W\left(\bar{V}_{1}\right) \Delta \bar{V}_{1}
\end{aligned}
$$

With (30), one has:

$$
\Delta V \leq \frac{b}{2 d}\left(\vartheta(k)-\vartheta^{*}\right)^{2}-\frac{\sigma}{2}\left(\vartheta^{*}-\vartheta(k)\right)^{2}+\frac{\sigma}{2}\left(\vartheta^{*}\right)^{2}-b V_{2}-a \bar{V}_{1} W\left(\bar{V}_{1}\right)
$$

where $\int_{0}^{\bar{V}_{1}} W(\sigma) d \sigma \leq W\left(\bar{V}_{1}\right) \bar{V}_{1}$ and $\sigma d>b$, such that $\Delta V \leq \frac{\sigma}{2}\left(\vartheta^{*}\right)^{2}-\bar{b} V$ with $\bar{b}=\min \{b, a\}$, one has:

$$
V(k) \leq \frac{\sigma}{2 \bar{b}}\left(\vartheta^{*}\right)^{2}+e^{-\bar{b} k} V(0)
$$

Since $W(0) \bar{V}_{1} \leq \int_{0}^{\bar{V}_{1}} W(\sigma) d \sigma$, combining (11), (19), (29) and (33), one has:

$$
\|\delta(k)\|^{2} \leq \frac{\sigma}{2 \eta_{\min }(P) W(0) \bar{b}}\left(\vartheta^{*}\right)^{2}+\frac{V(0)}{\eta_{\min }(P) W(0)} e^{-\overline{b k}},
$$

and

$$
\|z(k)\|^{2} \leq \frac{\sigma}{2 \bar{b}}\left(\vartheta^{*}\right)^{2}+e^{-\bar{b} k} V(0)
$$

With the following inequality:

$$
\begin{aligned}
\|x(k)\|^{2} & =\left\|x_{1}(k)\right\|^{2}+\left\|x_{2}(k)\right\|^{2} \\
& \leq\left\|E^{-1}\right\|^{2}\left\|z(k)+\mathrm{C}_{l} \zeta(k)+\mathrm{D}_{l} y_{1}(k)\right\|^{2}+\left\|x_{1}(k)\right\|^{2} \\
& \leq 3\left\|E^{-1}\right\|^{2}\|z(k)\|^{2}+\left\|x_{1}(k)\right\|^{2} \\
& +3\left\|E^{-1}\right\|^{2}\left\|D_{l} \tilde{C}\right\|^{2}\left\|x_{1}(k)\right\|^{2}+3\left\|E^{-1}\right\|^{2}\left\|\mathrm{C}_{l}\right\| \zeta(k) \|^{2} \\
& \leq 3\left\|E^{-1}\right\|^{2}\|z(k)\|^{2}+v\|\delta(k)\|^{2}
\end{aligned}
$$

where $v=\max \left\{3\left\|E^{-1}\right\|^{2}\left\|C_{l}\right\|, \quad 3\left\|E^{-1}\right\|^{2}\left\|D_{l} \tilde{C}\right\|^{2}+1\right\}$, one has:

$$
l_{2}+l_{1} e^{-\bar{b} k} \geq\|x(k)\|^{2}
$$

where

$$
l_{1}=3\left\|E^{-1}\right\|^{2} V(0)+\frac{v V(0)}{\eta_{\min }(P) \mathrm{W}(0)}
$$

$$
\begin{aligned}
& \Phi(\delta(k))+W\left(\bar{V}_{1}\right)\left(-c \bar{V}_{1}+\bar{\varepsilon}\|z(k)\|^{2}\right) \\
&\left.\leq-a \bar{V}_{1} W\left(\bar{V}_{1}\right)+W\left(\bar{V}_{1}\right)(a-c+\mu) \bar{V}_{1}+\bar{\varepsilon}\|z(k)\|^{2}\right) \\
& \leq-a \bar{V}_{1} W\left(\bar{V}_{1}\right)+\bar{\varepsilon} W\left(\frac{\bar{\varepsilon}}{c-a-\mu}\|z(k)\|^{2}\right)\|z(k)\|^{2}
\end{aligned}
$$

One knows, if $\bar{V}_{1} \leq \frac{\bar{\varepsilon}}{c-a-\mu}\|z(k)\|^{2}$, using $\frac{\bar{\varepsilon}}{c-a-\mu}\|z(k)\|^{2}$ instead of $\bar{V}_{1}$ in (32), and if $\bar{V}_{1}>\frac{\bar{\varepsilon}}{c-a-\mu}\|z(k)\|^{2}$, (32) also holds. Substituting (32) into (31) with $-\sigma\left(\vartheta(k)-\vartheta^{*}\right) \vartheta(k) \leq \frac{\sigma}{2}\left(\vartheta^{*}\right)^{2}-\frac{\sigma}{2}\left(\vartheta^{*}-\vartheta(k)\right)^{2}$, one has:
$l_{2}=\frac{3\left\|E^{-1}\right\|^{2} \sigma\left(\vartheta^{*}\right)^{2}}{2 \bar{b}}+\frac{v \sigma\left(\vartheta^{*}\right)^{2}}{2 \eta_{\min }(P) W(0) \bar{b}}$

With the above analysis, the system state $x(k)$ converges to bounded region $\Psi_{x}=\left\{x(k) \mid\|x(k)\|^{2} \leq l_{2}\right\}$. Then the proof is completed.

\section{Case Expansion}

Consider a nonlinear discrete system with multiple time-varying delays as follows:

$$
\left\{\begin{array}{l}
x(k+1)=f\left(x, x_{\tau 1} x_{\tau 2}, \ldots, x_{\tau r}\right)+g(x) u(k) \\
y(k)=h(x)
\end{array}\right.
$$

where $x(k) \in R^{n}$ is state vector, $u(k) \in \mathrm{R}^{m}$ and $y(k) \in R^{p}$ are the input and output of the system (35) with $m<p \leq n$. $f(\bullet), g(\bullet)$ and $h(\bullet)$ are the nonlinear functions with $f(0)=0, g(0)=0, h(0)=0$ and $\operatorname{Rank}[g(x)]=m$. $x_{\tau i}=x\left(k-\tau_{i}(k)\right)$ for $i \in[1, r]$, the time-varying delays parameter $\tau_{j}(k)$ satisfying $\tau_{j}(k) \leq \bar{\tau}_{j}$ and $\tau_{j}(k+1) \leq \tau_{j}^{*}<1$.

For the general nonlinear case (35), there exists a state transformation relation $\mathrm{T}(x)=[z, y]$ such that:

$$
\left\{\begin{array}{l}
z(k+1)=f_{1}\left(\bar{z}, y_{2}, \bar{z}_{\tau 1}, \bar{z}_{\tau 2}, \ldots, \bar{z}_{\tau r}, y_{2 \tau 1}, y_{2 \tau 2}, \ldots, y_{2 \tau r}\right) \\
y_{1}(k+1)=f_{2}\left(\bar{z}, y_{2}, \bar{z}_{\tau 1}, \bar{z}_{\tau 2}, \ldots, \bar{z}_{\tau r}, y_{2 \tau 1}, y_{2 \tau 2}, \ldots, y_{2 \tau r}\right) \\
y_{2}(k+1)=f_{3}\left(\bar{z}, y_{2}, \bar{z}_{\tau 1}, \bar{z}_{\tau 2}, \ldots, \bar{z}_{\tau r}, y_{2 \tau 1}, y_{2 \tau 2}, \ldots, y_{2 \tau r}\right) \\
\quad+\bar{g}(y) u(k) \\
y^{T}=\left[\begin{array}{ll}
y_{1}^{T} & y_{2}^{T}
\end{array}\right]
\end{array}\right.
$$

where $\bar{z}^{T}=\left[\begin{array}{ll}z^{T} & y_{1}^{T}\end{array}\right], f_{1}(\bullet), f_{2}(\bullet) \quad f_{3}(\bullet)$ and $\bar{g}(\bullet)$ are the nonlinear functions with the transformation.

The system (36) is rewritten as: 


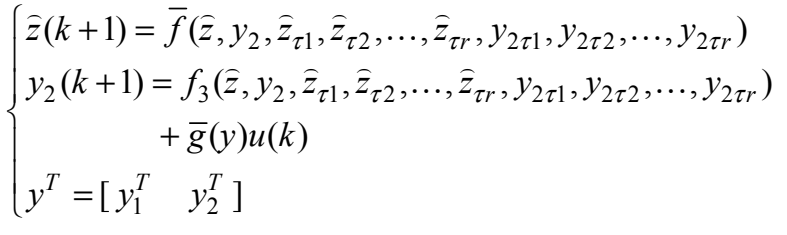

where $\bar{f}^{T}=\left[\begin{array}{ll}f_{1}^{T} & f_{2}^{T}\end{array}\right]$.

For the system (37), design the dynamic compensator as follows:

$$
\left\{\begin{array}{l}
\delta(k+1)=\Omega\left(\eta, y_{1}\right) \\
y_{2}^{*}(k)=\Theta\left(\eta, y_{1}\right)
\end{array}\right.
$$

where $y_{2}^{*}(k)$ is the subsidiary variable, $\Omega(\bullet)$ and $\Theta(\bullet)$ are the nonlinear functions. For the system (37) with the compensation controller (38), there exists a discrete Lyapunov-Krasovskii functional $V$ such that:

$$
\left\{\begin{array}{l}
\kappa(\|\hat{z}\|) \leq V \\
\alpha\left(\|\varsigma\|^{2}\right)-\Upsilon V \geq \Delta V
\end{array}\right.
$$

where $\hat{z}^{T}=\left[\begin{array}{ll}\bar{z}^{T} & \delta^{T}\end{array}\right], \varsigma=y_{2}-y_{2}^{*} . \kappa(\bullet)$ and $\alpha(\bullet)$ are the nonlinear functions with $\Upsilon$ is a positive scalar. Then, with $\varsigma=y_{2}-y_{2}^{*}$, one has:

$$
\begin{aligned}
\Delta \varsigma & =f_{3}-\Delta \Theta\left(\delta, y_{1}\right)+\bar{g}(y) u(t) \\
& =f_{3}-\left.\Delta \Theta\right|_{y_{1}} f_{2}-\left.\Delta \Theta\right|_{\delta} \Omega\left(\delta, y_{1}\right)+\bar{g}(y) u(k)
\end{aligned}
$$

For (40), the following Assumption 3. holds:

Assumption 3. For (40), there exist the nonlinear functions $f_{2}(\cdot)$ and $f_{3}(\cdot)$ such that:

$$
\left\{\begin{array}{l}
\sum_{j=0}^{r}\left(\Omega_{1 j}\left(\kappa\left\|\hat{z}_{\tau j}\right\|\right)+\Omega_{2 j}\left\|\varsigma_{\tau j}\right\|\right) \geq\left\|\left.\Delta \Theta\right|_{y_{1}} f_{2}\right\| \\
\sum_{j=0}^{r}\left(\vartheta_{1 j}^{T} \alpha_{1 j}\left(\kappa\left\|\hat{z}_{\tau j}\right\|\right)+\vartheta_{2 j}^{T} \alpha_{2 j}\left\|\varsigma_{\tau j}\right\|\right) \geq\left\|f_{3}\right\|
\end{array}\right.
$$

where $\vartheta_{1 j} \in R^{p_{1 j}}$ and $\vartheta_{2 j} \in R^{p_{2 j}}$ are the unknown constant vectors, $\Omega_{1 j}(\bullet)$ and $\Omega_{2 j}(\bullet)$ are the nonlinear function. $\left[\alpha_{i j}(\bullet)\right]^{T}=\left[\alpha_{i j 1}(\bullet), \alpha_{i j 2}(\bullet), \ldots, \alpha_{i j p_{i j}}(\bullet)\right]$ for $i=1,2$, there exist the functions $\bar{\alpha}_{1 j i}(\bullet), \bar{\alpha}_{2 j i}(\bullet)$ and $\bar{\Omega}_{2 j}(\bullet)$ such that $\alpha_{1 j i}^{2}(a) \leq a^{2} \bar{\alpha}_{1 j i}^{2}(a), \alpha_{2 j i}^{2}(a) \leq a^{2} \bar{\alpha}_{2 j i}^{2}(a)$ and $\Omega_{2 j}^{2}(a) \leq a^{2} \bar{\Omega}_{2 j}^{2}(a)$.

Theorem 3. For the system (36), there exists a dynamic compensator (38) satisfying (39), design the controller $u(k)=\bar{g}^{-1}(y) \bar{u}(k)$

where

$$
\begin{aligned}
\bar{u}(k)= & \left.\Delta \Theta\right|_{\delta} \Omega\left(\delta, y_{1}\right)-\frac{1}{2} \varsigma\left\|\bar{\alpha}_{20}(\|\varsigma\|)\right\|^{2}-\frac{1}{2} \varsigma_{\Omega_{2 j}}^{2}(\|\varsigma\|) \\
& -\frac{1}{2} \varsigma \sum_{j=1}^{r}\left(1-\tau_{j}^{*}\right)^{-1} e^{c \bar{\tau}_{j}}\left(\left\|\bar{\alpha}_{2 j}(\|\varsigma\|)\right\|^{2}+\bar{\Omega}_{2 j}^{2}(\|\varsigma\|)\right) \\
& -\frac{1}{2} \varsigma \bar{\alpha}\left(\|\varsigma\|^{2}\right) W\left(\frac{\alpha\left(\|\varsigma\|^{2}\right)}{\Upsilon-\mu-c}\right)-\frac{1}{2}(c+\vartheta(k)) \varsigma
\end{aligned}
$$

in which $\mu$ and $c$ are the positive scalars satisfying $0<\Upsilon-\mu-c, W(\bullet)$ is a positive increasing function satisfying (43). The adaptive law is designed as $\vartheta(k+1)=d\|\varsigma(k)\|^{2}-\sigma d \vartheta(k)$ with $d>0, \sigma>0$ and $\sigma d>c$, then the solutions of the close-loop system converge to a bounded region.

Proof. Consider the Lyapunov-Krasovskii functional as follows:

$$
U_{F}=U_{F 1}+U_{F 2}
$$

where

$$
\begin{gathered}
U_{F 1}=\varsigma^{T} \varsigma+\int{ }_{0}^{V} W(w) \mathrm{d} w+\frac{1}{2 d}\left(\vartheta(k)-\vartheta^{*}\right)^{2}, \\
U_{F 2}=\sum_{j=1}^{r}\left(1-\tau_{j}^{*}\right)^{-1} e^{c \bar{\tau}_{j}} \int_{k-\tau_{j}(k)}^{k} e^{c(\delta-k)} \times\left(\left\|\alpha_{1 j}(\kappa(\|\hat{z}(w)\|))\right\|^{2}\right. \\
\left.+\left\|\alpha_{2 j}(\|\varsigma(w)\|)\right\|^{2}+\Omega_{1 j}^{2}(\kappa(\|\hat{z}(w)\|))+\Omega_{2 j}^{2}(\|\varsigma(w)\|)\right) d w
\end{gathered}
$$

in which $c$ is a scalar, $\vartheta^{*}=\sum_{j=0}^{r}\left(2+\vartheta_{1 j}^{T} \vartheta_{1 j}+\vartheta_{2 j}^{T} \vartheta_{2 j}\right)$.

Via the same way, one has

$$
\Delta U_{F} \leq \frac{\sigma}{2}\left(\vartheta^{*}\right)^{2}-c U_{F 2}-c U_{F 1} \leq \bar{\sigma}-c U_{F}
$$

where $\bar{\sigma}=\frac{\sigma\left(\vartheta^{*}\right)^{2}}{2}$.

\section{Simulation Example}

Consider the nonlinear discrete multiple time-delays mobile robot system as follows:

$$
\left\{\begin{aligned}
q(k+1)= & \left(A_{g 0}+\Delta A_{g 0}\right) q(k)+A_{g 1} q\left(k-\tau_{1}(k)\right) \\
& +A_{g 2} q\left(k-\tau_{2}(k)\right)+B_{g}(u(k)+f) \\
y_{q}(k)= & C_{g} q(k)
\end{aligned}\right.
$$

where $q(k) \in R^{3}, u(k) \in R^{2}$ and $y_{q}(k) \in R^{3}$ are the state vector, control input and output of the mobile robot, respectively. $A_{g 0} \in R^{3 \times 3}, A_{g 1} \in R^{3 \times 3}, A_{g 2} \in R^{3 \times 3}, B \in R^{3 \times 2}$, and $C \in R^{3 \times 3}$ are the known matrices. $\Delta A_{g 0}$ is an unknown matrix representing the parametric uncertainty. where 
$q(k)=\left[\begin{array}{lll}q_{x}(k) & q_{y}(k) & q_{\theta}(k)\end{array}\right]^{T}, u(k)=\left[\begin{array}{lll}v_{q}(k) & \omega_{p}(k)\end{array}\right]^{T}, q_{x}(k)$ and $q_{y}(k)$ are the robot position coordinate, $q_{\theta}(k)$ is the robot direction angle, $v_{p}(k)$ and $\omega_{p}(k)$ are the linear velocity and angular velocity of the robot, respectively.

$$
A_{g 0}=\left[\begin{array}{ccc}
0.8 & 0.8 & 0 \\
1 & 0.5 & 2 \\
2 & 1 & 0.5
\end{array}\right], A_{g 1}=\left[\begin{array}{ccc}
0.5 & 0 & 0.5 \\
1 & 2 & 2 \\
1 & 0.6 & 0.6
\end{array}\right], A_{g 2}=\left[\begin{array}{ccc}
0 & 0 & 1 \\
1 & 1 & 0 \\
1 & 2 & 0.8
\end{array}\right], B_{g}=\left[\begin{array}{ll}
0 & 0 \\
1 & 0 \\
0 & 1
\end{array}\right] \text { and } C_{g}=\left[\begin{array}{lll}
0 & 0 & 0 \\
0 & 1 & 0 \\
0 & 0 & 1
\end{array}\right]
$$

where $f^{T}=\left[f_{2}, f_{2}\right]$,

in which

$$
\begin{aligned}
& f_{1}=\chi_{1} q^{T}(k) q\left(k-\tau_{1}(k)\right)+\chi_{2} q^{T}\left(k-\tau_{1}(k)\right) q\left(k-\tau_{2}(k)\right) \\
& f_{2}=\chi_{2} q^{\mathrm{T}}\left(k-\tau_{1}(k)\right) q\left(k-\tau_{2}(k)\right)+\chi_{3} q^{T}(k) q\left(k-\tau_{2}(k)\right)
\end{aligned}
$$

where $\chi_{i}$ are the unknown scalars, $\tau_{1}$ and $\tau_{2}$ are the time-delays.

For the system (43), design $E_{1}=\left[\begin{array}{lll}0.5 & 1 & -0.5\end{array}\right]^{T}, E_{2}=0.65, \lambda_{1}=\operatorname{diag}\{0.6,0.6,0.6\}, \lambda_{2}=\operatorname{diag}\{1,1\}$ and $M(k)^{2} \leq 1$.

Now, employing the proposed method in this study to construct the output feedback controller. The matrices $A_{l}, B_{l}, C_{l}$ and $D_{l}$ are given as follows

$$
\left[B_{l}, A_{l}\right]=\left[\begin{array}{ccc}
0.1125 & -5.9700 & 1.3100 \\
-1.9980 & 0.1683 & -9.8650
\end{array}\right],\left[D_{l}, \mathrm{C}_{l}\right]=\left[\begin{array}{ccc}
0.9430 & 0.3128 & 0.0455 \\
-0.5025 & 0.3990 & 0.1600
\end{array}\right]
$$

With the Theorems 1 and 2, the following inequalities hold:

$$
\begin{aligned}
& \left|f_{1}\right| \leq \frac{1}{2}\left(\chi_{1}+\chi_{2}\right)\left\|q\left(k-\tau_{1}(k)\right)\right\|^{2}+\frac{1}{2} \chi_{2}\left\|q\left(k-\tau_{2}(k)\right)\right\|^{2}+\frac{1}{2} \chi_{1}\|q(k)\|^{2} \\
& \left|f_{2}\right| \leq \frac{1}{2}\left(\chi_{2}+\chi_{3}\right)\left\|q\left(k-\tau_{2}(k)\right)\right\|^{2}+\frac{1}{2} \chi_{2}\left\|q\left(k-\tau_{1}(k)\right)\right\|^{2}+\frac{1}{2} \chi_{3}\|q(k)\|^{2}
\end{aligned}
$$

With the Theorems 2 and 3, designing the controller as follows:

$$
\begin{aligned}
u(k)= & \mathrm{C}_{l}\left(A_{l} \zeta(k)+B_{l} y_{1}(k)\right)-\left(\bar{A}_{022}-D_{l} \tilde{C} \bar{A}_{012}\right) \times(z(k) \\
& \left.+C_{l} \zeta(k)\right)-\frac{1}{2} \vartheta(k) z(k)-13.8 z(k)-3\|z(k)\|^{2} z(k)
\end{aligned}
$$

with the adaptive law $\vartheta(k+1)=-6.9 \vartheta(k)+\|z(k)\|^{2}$.

For the simulations, the initial state of the robot system is $q=\left[\begin{array}{lll}0 & -1.25 & 2\end{array}\right]^{T}$. With the Assumption 2, the final value of $J^{*}$ is chosen as 18.5470 .

With the Lemma 2, design the parameters as follows:

$$
n_{1}=\left[\begin{array}{lll}
1 & 0 & 0 \\
0 & 0 & 0 \\
0 & 0 & 0
\end{array}\right], n_{2}=\left[\begin{array}{lll}
1 & 0 & 0 \\
0 & 0 & 0 \\
0 & 0 & 0
\end{array}\right] \text { and } \Theta=\Theta^{T}=\left[\begin{array}{lll}
1 & 0 & 0 \\
0 & 1 & 0 \\
0 & 0 & 1
\end{array}\right] .
$$

Here, design the cost function parameters as follows:

$$
A_{c}=\left[\begin{array}{ccc}
-0.2545 & -0.3013 & -0.4589 \\
0.1528 & 0.2354 & -0.0505 \\
0.1466 & 0.2597 & -0.4267
\end{array}\right], B_{c}=\left[\begin{array}{cc}
1.448 & 0.4306 \\
0.2577 & -0.0580 \\
-0.4313 & -3.1592
\end{array}\right], C_{c}=\left[\begin{array}{ccc}
-0.1246 & 0.3255 & -5.3794 \\
0.74 & -0.5897 & 0.4890 \\
-0.1246 & -0.0946 & 0.2246
\end{array}\right]
$$

The state responses are shown in Figures 1 and 2. The control input is shown in Figure 3. From the three figures, it can be seen that the proposed method is effective and can stabilize the mobile robot system quickly. 


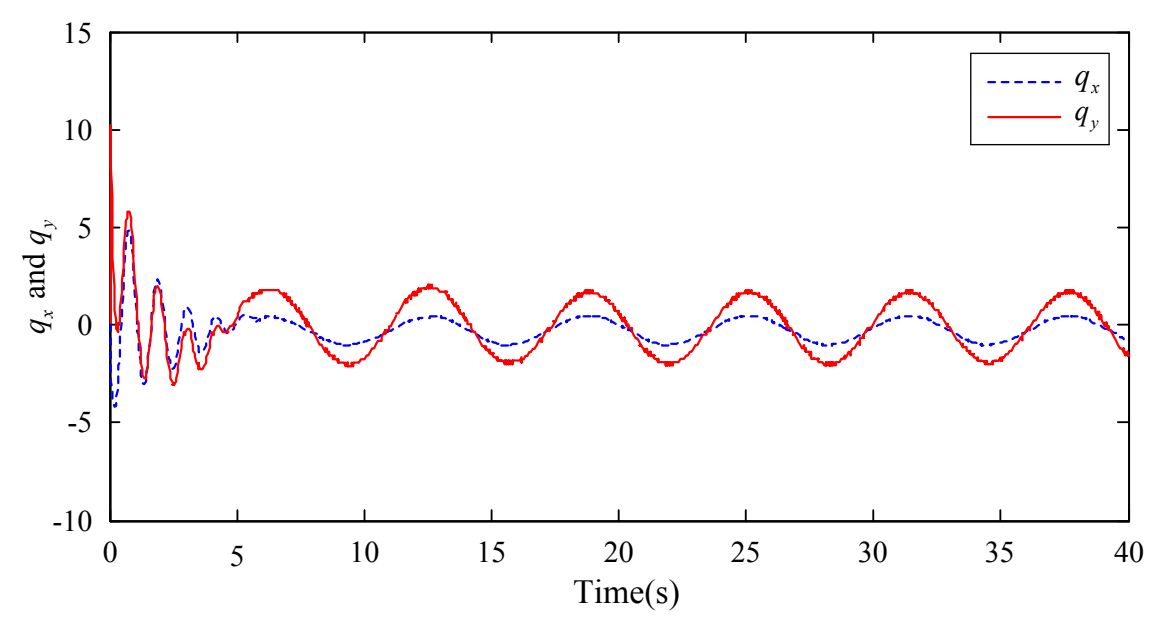

Figure 1. The responses of system state variables $q_{x}$ and $q_{y}$.

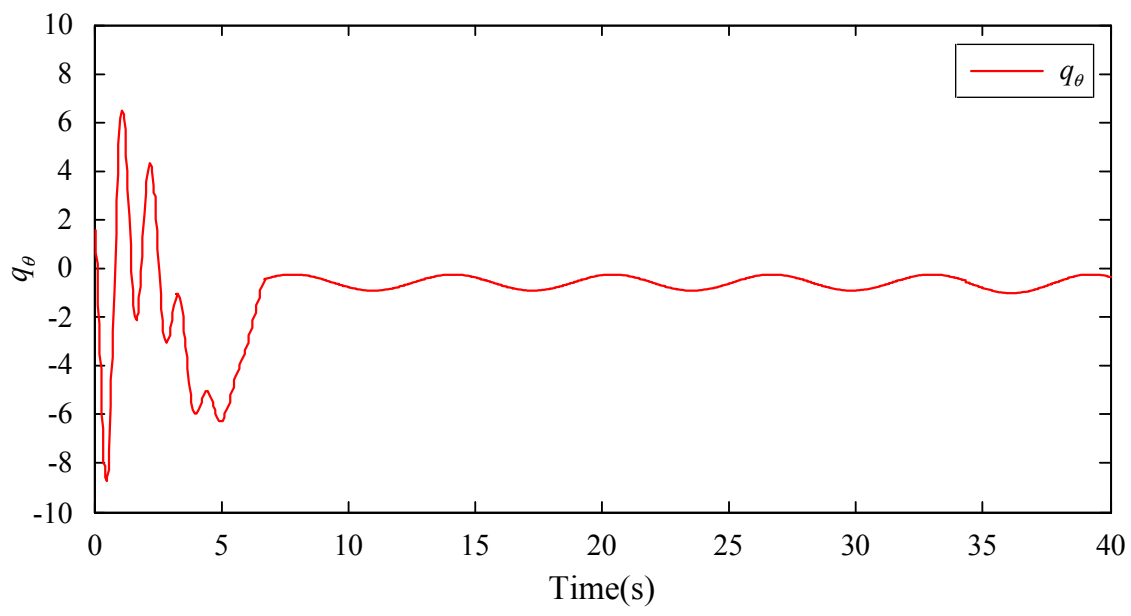

Figure 2. The response of system state variable $q_{\theta}$.

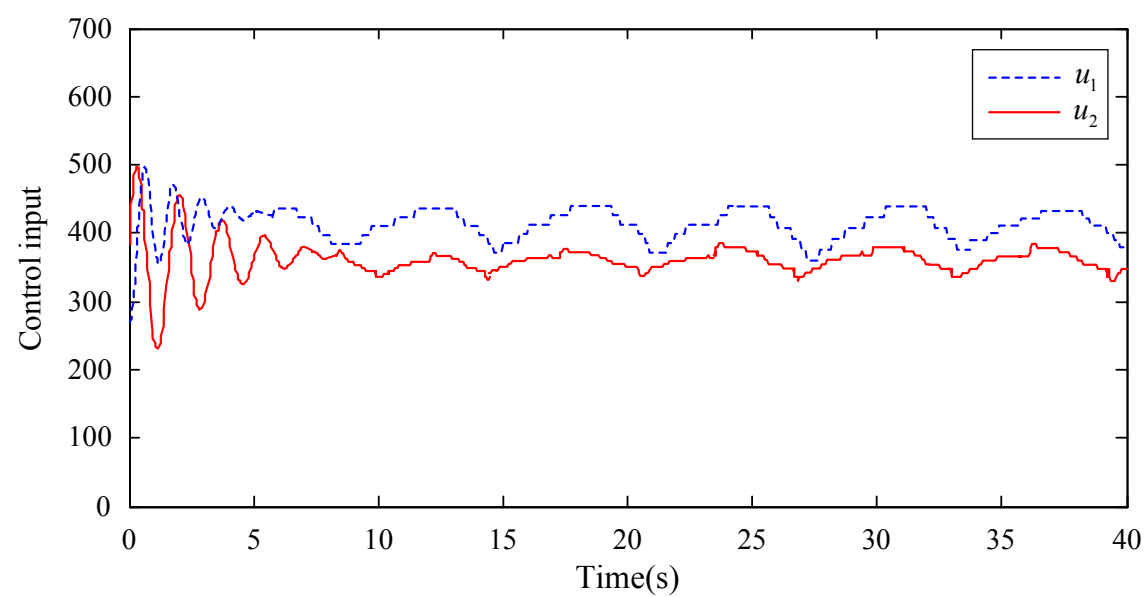

Figure 3. The responses of system control inputs.

\section{Conclusions}

This paper addressed the dynamic output feedback control problem for a class of nonlinear system with multiple time-varying delay and parametric uncertainties. The nonlinear uncertainties are in the nonlinear form and bounded by nonlinear functions with gains unknown. The dynamic compensator is designed and the control design condition is relaxed. The dynamic output feedback controller is constructed such that the solutions of the closed-loop system converge to an adjustable bounded region. The result is further extended to the general nonlinear case. Finally, the simulations for the mobile robot are performed and the results demonstrate the effectiveness of the proposed method. 


\section{Disclosure Statement}

No potential conflict of interest was reported by the authors.

\section{Funding}

The authors wish to thank the editors and the anonymous reviewers for their valuable suggestions and critical comments to improve the paper. This research was financially supported by National Natural Science Foundation of China (Project No. 61473248); Natural Science Foundation of Hebei Province (Project No. F2016203496); National Intellectual Property Office of China (Grant No. ZL-2012-1-0052200.2; Zl-2012-1-0052199.3; Zl-2014-2-0411083.9); and China National Heavy Machinery research institute.

\section{References}

[1] M. Luo, C. S. Li, X. Y. Zhao, R. H. Li and X. L. An "Compound feature selection and parameter optimization of ELM for fault diagnosis of rolling element bearings." Isa Transactions 65 (2016): 556.

[2] C. S. Li, J. Z. Zhou, B. Fu. P. G. Kou and J. Xiao. "T-S Fuzzy Model Identification With a Gravitational Search-Based Hyperplane Clustering Algorithm." IEEE Transactions on Fuzzy Systems 20.2 (2012): 305-317.

[3] C. S. Li, J. Z. Zhou, P. G. Kou and J. Xiao. "A novel chaotic particle swarm optimization based fuzzy clustering algorithm." Neurocomputing 83.15 (2012): 98-109.

[4] C. C. Hua, Q. G. Wang, and X. P. Guan. "Adaptive fuzzy output-feedback controller design for nonlinear time-delay systems with unknown control direction." IEEE Transactions on Systems Man \& Cybernetics Part B Cybernetics 39.2 (2005): 363-374.

[5] J. Wei, Y. Zhang, M. Sun, and B. Geng. "Adaptive iterative learning control of a class of nonlinear time-delay systems with unknown backlash-like hysteresis input and control direction." Isa Transactions (2017). DOI: 10.1016/j.isatra.2017.05.007.

[6] L. Jing, and H. K. Khalil. "High-gain-predictor-based output feedback control for time-delay nonlinear systems." Automatica 71 (2016): 324-333.

[7] O. M. Kwon, H. P. Ju, S. M. Lee, and E. J. Cha. "New augmented Lyapunov-Krasovskii functional approach to stability analysis of neural networks with time-varying delays." Nonlinear Dynamics 76.1 (2014): 221-236.

[8] I. V. Medvedeva, and A. P. Zhabko. "Synthesis of Razumikhin and Lyapunov-Krasovskii approaches to stability analysis of time-delay systems." Automatica 51 (2015): 372-377.

[9] Éva Gyurkovics. "Guaranteed cost control of discrete-time uncertain systems with both state and input delays." International Journal of Control 23.5 (2016): 1-13.

[10] Z. Wang, B. Shen, H. Shu, and G. Wei. "Quantized H- $\infty$ control for nonlinear stochastic time-delay systems with missing measurements." IEEE Transactions on Automatic Control 57.6 (2012): 1431-1444.

[11] Q. Zhou, P. Shi. S. Xu, and H. Li. "Adaptive static output feedback control for nonlinear time-delay systems by fuzzy approximation approach." IEEE Transactions on Fuzzy Systems 21.2 (2013): 301-313.

[12] F. Z. Gao, and Y. Q. Wu. "Global stabilisation for a class of more general high-order time-delay nonlinear systems by output feedback." International Journal of Control 88.8 (2015): 1540-1553.

[13] S. C. Tong, and Y. Li. "Adaptive fuzzy output feedback tracking back-stepping control of strict-feedback nonlinear systems with unknown dead zones." IEEE Transactions on Fuzzy Systems 20.1 (2012): 168-180.

[14] S. Y. Liu, Y. Liu, and N. Wang. "Nonlinear disturbance observer-based back-stepping finite-time sliding mode tracking control of underwater vehicles with system uncertainties and external disturbances." Nonlinear Dynamics (2016): 1-12.

[15] Y. H. Choi, and S. J. Yoo. "Minimal-approximation-based decentralized back-stepping control of interconnected time-delay systems." IEEE Transactions on Cybernetics (2016): 1-13.

[16] D. Huang, and S. K. Nguang. "State feedback control of uncertain networked control systems with random time delays." IEEE Transactions on Automatic Control 53.3 (2008): 829-834.

[17] Y. T. Wang, X. Lin, and X. Zhang. "State feedback stabilization for neutral-type neural networks with time-varying discrete and unbounded distributed delays." Journal of Control Science \& Engineering 2012.1 (2012): 966-989.

[18] H. Q. Wang, P. X. Liu, and S. Peng. "Observer-based fuzzy adaptive output-feedback control of stochastic nonlinear multiple time-delay systems." IEEE Transactions on Cybernetics 99.5 (2017): 1-11.

[19] M. Wang, B. Chen, K. Liu, X. Liu, and S. Zhang. "Adaptive fuzzy tracking control of nonlinear time-delay systems with unknown virtual control coefficients." Information Sciences 178.22 (2015): 4326-4340.

[20] X. J. Xie, and L. Liu. "Further results on output feedback stabilization for stochastic high-order nonlinear systems with time-varying delay." Automatica 48.10 (2012): 2577-2586.

[21] T. Dierks, and S. Jagannathan. "Neural network output feedback control of robot formations." IEEE Transactions on Systems Man \& Cybernetics Part B Cybernetics A Publication of the IEEE Systems Man \& Cybernetics Society 40.2 (2016): 383-393.

[22] B. Ren, S. S. Ge, K. P. Tee, and T. H. Lee. "Adaptive neural control for output feedback nonlinear systems using a barrier Lyapunov function." IEEE Transactions on Neural Networks 21.8 (2015): 1339.

[23] J. Z. Peng, Y. Liu, and J. Wang. "Fuzzy adaptive output feedback control for robotic systems based on fuzzy adaptive observer." Nonlinear Dynamics 78.2 (2016): 789-801.

[24] H. Yue, and J. Li. "Output-feedback adaptive fuzzy control for a class of non-linear time-varying delay systems with unknown control directions." IET Control Theory \& Applications 6.9 (2016): 1266-1280.

[25] C. C. Hua, L. Zhang, and X. Guan. "Decentralized output feedback controller design for nonlinear interconnected systems with unknown control direction and time-varying delays." International Journal of Adaptive Control \& Signal Processing 28.11 (2014): 1160-1173. 
[26] Y. Li, and F. Gaob. "Optimal guaranteed cost control of discrete -time uncertain systems with both state and input delays." Journal of the Franklin Institute 338.1 (2016): 101-110.

[27] M. S. Mahmoud. Resilient control of uncertain dynamical systems. Springer, Berlin, Heidelberg, (2014).

[28] Y. He, G. P. Liu, D. Rees, and M. Wu. "Improved stabilisation method for networked control systems." IET Control Theory \& Applications 1.6 (2007): 1580-1585.

[29] Y. He, M. Wu, G. P. Liu, and J. H. She. "Output feedback stabilization for a discrete-time system with a time-varying delay." IEEE Transactions on Automatic Control 53.10 (2016): 2372-2377. 